\title{
EL BIODERECHO: RELACIÓN ENTRE LA BIOÉTICA Y LOS DERECHOS HUMANOS
}

THE BIOLAW: RELATIONSHIP BETWEEN BIOETHICS AND HUMAN RIGHTS

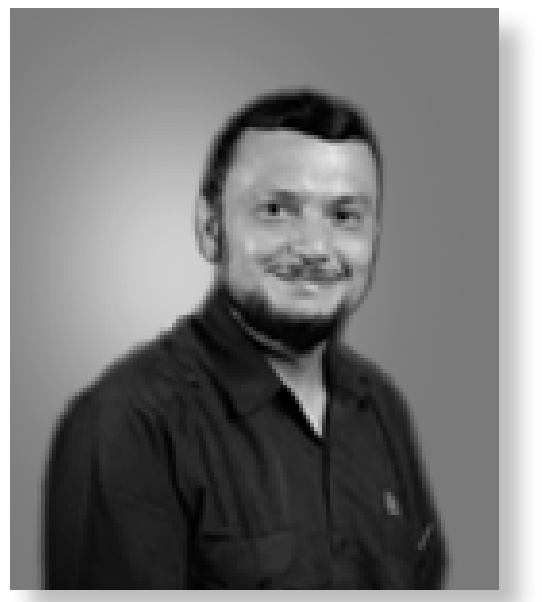

Alejandro Rosillo Martínez

Doctor en Derechos Humanos por la Universidad Carlos III de Madrid

alejandro.rosillo@uaslp.mx

México

\section{COMPRENSIÓN DEL "DERECHOS HUMANOS"}

En términos generales, "derechos humanos" es un concepto moderno que se refiere a varias facetas de la vida política de las sociedades construidas bajo este imaginario. Estas podrían resumirse en:

a) Una comprensión de la ética pública: La acción política está en función del cumplimiento de ciertos principios éticos que se encuentran plasmados en los derechos humanos; de ahí que se relacione a éstos con la dignidad humana, por su contenido ético fundamental. Se insiste que los derechos humanos son la expresión jurídico-política de una ética mínima que guía la acción del Estado y la convivencia de los gobernados, que permite además que cada persona pueda realizar su propio proyecto de vida.

b) Una comprensión de la función del Estado: El Estado se constituye como un "Estado de Derecho", es decir, que sus acciones deben sustentarse en el cumplimiento de la ley. La legalidad es la base del ejercicio del poder del Estado, que le significa un límite y una pauta de acción, tanto para no realizar aquello que tiene prohibido o para no omitir aquello que tiene como mandato. En efecto, no cualquier ley justifica el Estado de Derecho, sino debe ser una ley acorde con el contenido de los derechos humanos. En este sentido, Luigi Ferrajoli distingue entre la legalidad en sentido lato y la legalidad en sentido estricto:

a la legalidad en sentido lato, o validez formal, que exige solamente que sean predeterminados por ley los sujetos titulares y las formas de ejercicio de todo poder; $y$ a la legalidad en sentido estricto, o validez sustancial, que requiere además que estén legalmente preordenadas $\mathrm{y}$ circunscritas, mediante obligaciones $\mathrm{y}$ prohibiciones, las materias de competencia y los criterios de decisión. (Ferrajoli, 2009, p. 856).

c) Una comprensión de legitimidad de la acción del individuo: Los derechos humanos conformarían una "esfera" de actuación legítima del individuo en relación con los otros individuos y con el Estado. Por un lado, cada individuo es titular de una esfera de derechos que le significan tanto el ámbito legítimo de su accionar como los límites de éste, es decir, "mis derechos terminan donde empiezan los derechos del otro". Por otro lado, los individuos tienen una esfera de protección ante la acción del poder del Estado, 
tanto en sentido negativo (por ejemplo, el Estado no puede impedirme expresar mis ideas) como positivo (por ejemplo, tengo la expectativa de recibir un servicio de educación por parte del Estado).

Algunas de estas facetas han sido contenidas en otras expresiones que guardan relación con el concepto "derechos humanos". Estas son: derechos naturales, derechos subjetivos públicos, libertades públicas, derechos morales, garantías individuales, derechos fundamentales (Peces-Barba, 1999, pp. 21-38; Ramírez García, 2014, pp. 23-29). Cada una de estas acepciones se ha construido en diversos contextos y son productos de distintos procesos; además, resaltan algún aspecto de eso que en general se ha llamado "derechos humanos". En este contexto, una comprensión básica ${ }^{1}$ del concepto de "derechos humanos" parte de entenderlos como derechos subjetivos; por ejemplo, una definición que se ha vuelto clásica es la otorgada por Luigi Ferrajoli:

Son "derechos fundamentales" todos aquellos derechos subjetivos que corresponden universalmente a 'todos' los seres humanos en cuanto dotados del status de personas, de ciudadanos o personas con capacidad de obrar; entendiendo por 'derecho subjetivo' cualquier expectativa positiva (de prestaciones) o negativa (de no sufrir lesiones) adscrita a un sujeto por una norma jurídica; y por 'status' la condición de un sujeto, prevista asimismo por una norma jurídica positiva, como presupuesto de su idoneidad para ser titular de situaciones jurídicas y/o autor de los actos que son ejercicio de éstas. (Ferrajoli, 2001, p.19).

Ahora bien, ¿qué se entiende por derechos subjetivos? Como puede observarse en la definición citada, Ferrajoli lo comprende como una expectativa positiva (recibir una prestación) o una expectativa negativa (de no ser lesionado). Pero, ¿quién está obligado a cumplir con esta expectativa, sea positiva o negativa? Tratándose de la concepción general de derechos humanos ${ }^{2}$, es el Estado. Por lo tanto, se está ante la estructura más básica de un derecho subjetivo: una relación jurídica donde un sujeto tiene una expectativa (positiva o negativa) y otro sujeto tiene la obligación de cumplir con esta expectativa. Es decir, el derecho subjetivo se basa en una relación jurídica básica: a cada derecho -donde un sujeto A es titular- le corresponde una obligación -donde un sujeto B es titular-.

No obstante, como lo ha demostrado Wensley Newcomb Hohfeld (1991), el concepto jurídico de "derecho subjetivo" no puede reducirse solo a la relación de derechos y obligaciones, ya que no todas las relaciones jurídicas a las que se refiere comprenden las mismas clases de acciones por parte de los sujetos implicados. Por eso, Hohfeld propone el análisis del derecho subjetivo a partir de las diversas posiciones que los sujetos titulares y obligados se encuentra. No realiza una definición formal, más bien presenta dos esquemas: los correlativos y los opuestos.

Los correlativos se refieren a las cuatro relaciones entre el sujeto titular (A) y el sujeto obligado (B). Estos serían:
a. Cuando A tiene una pretensión (o derecho, estrictamente hablando), B tiene un deber.
b. Cuando A tiene una libertad, B tiene un no- derecho.
c. Cuando A tiene una competencia, B tiene una sujeción.
d. Cuando A tiene una inmunidad, B tiene una incompetencia.

Por otro lado, los opuestos jurídicos se refieren al contenido de cada posición:
a. Una pretensión (o derecho, estrictamente hablando) es lo contrario a un no-derecho.
b. Una libertad es lo contrario a un deber.
c. Una competencia es lo contrario a una incompetencia.
d. Una inmunidad es lo contrario a una sujeción.

Lo importante de esta clasificación para el tema de este artículo, es caer en la cuenta de las diversas situaciones que pueden referirse cuando se dice que alguien tiene un derecho. En efecto, los derechos humanos son derechos subjetivos que, buscando promover la dignidad humana, colocan como obligado -principal, aunque no exclusivamente- al Estado y como titular a la persona gobernada.

\section{CONSTITUCIONALISMO Y DERECHOS HUMANOS}

Los Estados modernos suelen clasificarse como Estados constitucionales, debido a que asumen que el texto constitucional es la norma suprema. Las estructuras jurídicas, políticas y administrativas de

1 El abordaje filosófico sobre los derechos humanos suele dividirse en dos grandes temas: el concepto y el fundamento. Analizar estos temas rebasa por mucho el objetivo de este artículo. Sólo se pretende presentar una comprensión básica, que si bien simplificada y ubicada en el ámbito jurídico, nos sirve para presentar una de las conexiones que el derecho tiene con la bioética. Para profundizar estos temas, puede verse: Rosillo Martínez, 2013; Herrera Flores, 2005; Sanchez Rubio, 2018.

2 Un problema importante en la materia de derechos humanos es la eficacia horizontal de ellos. Es decir, cuando el obligado a cumplir un derecho humano, y por lo tanto, quien puede violarlo, no es el Estado sino otro tipo de sujeto, que se llamaría "particular". No se profundizară al respecto, pero es importante tener en cuenta esta cuestión ya que, en el Bioderecho, muchos obligados a cumplir los derechos humanos de un paciente, por ejemplo, no tienen que ser forzosamente parte del Estado. Al ser la protección de la salud una obligación que corresponde inicialmente al Estado -ya sea proporcionando directamente un servicio o generando una regulación al respecto-, cuando se delega a privados, éstos también son obligados en materia de derechos humanos. 
un Estado se encuentran en un texto constitucional. Este texto es producto de un "poder constituyente" que a través de la Constitución crea los "poderes constituidos". De ahí que estos textos constitucionales tengan una "parte orgánica", que refiere la organización de las instituciones que conforman el Estado.

Otra sección de los textos constitucionales modernos es donde se reconocen los derechos humanos o derechos fundamentales, que suele llamarse "parte dogmática". En ella aparece el catálogo, más o menos amplio, de derechos y bienes que prioritariamente deben ser protegidos por el Estado; como se decía líneas arriba, se establecen los fines que justifican la existencia y la acción de las instituciones y poderes estatales.

En junio de 2011, se realizó una reforma a la Constitución Política de los Estados Unidos Mexicanos (CPEUM) en materia de derechos humanos. Entre otros artículos, modificó el artículo $1^{\circ}$ que estableció como normas superiores del ordenamiento jurídico a los derechos humanos contenidos en la Constitución y en los tratados internacionales de la materia (conocido como el "bloque de constitucionalidad"), en los siguientes términos:

Artículo 1o. En los Estados Unidos Mexicanos todas las personas gozarán de los derechos humanos reconocidos en esta Constitución y en los tratados internacionales de los que el Estado Mexicano sea parte, así como de las garantías para su protección, cuyo ejercicio no podrá restringirse ni suspenderse, salvo en los casos $\mathrm{y}$ bajo las condiciones que esta Constitución establece.

También establece el principio propersona y la interpretación conforme, en el segundo párrafo:

Las normas relativas a los derechos humanos se interpretarán de conformidad con esta Constitución y con los tratados internacionales de la materia favoreciendo en todo tiempo a las personas la protección más amplia.

Por su parte, el artículo 133 de la CPEUM establece el principio de la supremacía constitucional, que no es otra cosa que la norma jurídica que contempla la jerarquía de normas en el sistema mexicano. En una primera lectura, parecería que este artículo establece la superioridad del derecho federal sobre el derecho de los Estados. Sin embargo, esto violentaría el propio sistema federal, ya que cada Estado es libre y soberano en función de las facultades que le otorga la Constitución. Por eso, la interpretación más aceptada versa en que este artículo hace referencia a la supremacía constitucional, es decir, en que ni las leyes emanadas de la federación ni las leyes emanadas de los estados pueden violentar la norma fundamental, que es la Constitución.

El principio de "supremacía constitucional" significa que la Constitución de un Estado es la norma de mayor jerarquía. En efecto, todas las normas que integran el sistema jurídico deben estar acordes con la norma fundamental, de forma que, si un tratado, una ley, un reglamento o una sentencia fuera contrario a lo establecido en la Constitución, entonces deberá declararse inválida esa norma por ser inconstitucional $\mathrm{o}$, en otras palabras, por ser un elemento que rompe la coherencia del sistema. Pero hay que tomar en cuenta que esta supremacía constitucional se ha complejizado en función de los ya mencionados elementos introducidos en la reforma de 2011: el bloque de constitucionalidad y el principio propersona. Estos son temas amplios y complejos, pero en función del objetivo de este texto se mencionan sus características generales.

El concepto "bloque de constitucionalidad" se refiere a la existencia de un conjunto de normas que tienen jerarquía constitucional en el ordenamiento jurídico. Es decir, se asume que no todas las normas con carácter constitucional se encuentran en el texto de la Constitución, sino que hay otras que el mismo texto constitucional hace la remisión. En la CPEUM existe una serie de artículos que hacen referencia a los tratados internacionales -en especial aquellos cuya materia es derechos humanos- como parte de las normas constitucionales. Se pueden mencionar los artículos $1^{\circ}, 15,103$ fracción I, 105 fracción II inciso g), 107 y 133. En efecto, el sistema jurídico mexicano reconoce un bloque de constitucionalidad, como se dijo, compuesto por las normas contenidas en la CPEUM y los derechos humanos establecidos en los tratados internacionales que México forme parte.

Por otra parte, el principio propersona se encuentra en el artículo $1^{\circ}$, segundo párrafo, de la CPEUM. Este principio conlleva diversas posibilidades:

a) Preferencia interpretativa: Se debe buscar la interpretación de la norma que mejor proteja el derecho humano en cuestión. En efecto, se dan diversas opciones:

- En caso de protección, se debe optar por la interpretación que más amplíe el goce del derecho.

- En caso de limitación, se debe optar por la interpretación que restrinja más las limitaciones para el ejercicio del derecho.

b) Preferencia de normas: En caso de existir dos o más normas que puedan aplicarse, se debe aplicar la norma más favorable a la persona, con 
independencia de la jerarquía formal de aquélla. Con lo anterior, puede verse, que la jerarquía de las normas jurídicas sufre una modificación, pues si bien sigue operando la supremacía constitucional, ésta se ve afectada por la existencia de un bloque de constitucionalidad y el principio propersona. En efecto, la mayor jerarquía la tendrían las normas del bloque de constitucionalidad y las normas que respondan a la aplicación del principio propersona. Con esto, se entiende que se está en una conjunción de Constitucionalismo y derechos humanos, al ubicar como las normas superiores de un sistema jurídico a los derechos fundamentales que se encuentran referidos en la Constitución (texto constitucional más tratados internacionales).

\section{DERECHOSFUNDAMENTALES:PRINCIPIOS O REGLAS}

La discusión sobre la manera en que los derechos fundamentales deben estar estructurados en un texto constitucional, ha sido bastante difundida: si como reglas o como principios. Entre las diversas problemáticas que esto acarrea, interesan dos: si realmente se puede defender una diferencia entre las normas jurídicas llamadas principios y las llamadas reglas, y cuál debería ser la estructura más adecuada para los derechos fundamentales.

Para las corrientes que se engloban en el neoconstitucionalismo ${ }^{3}$, en términos generales, los derechos fundamentales son la base del sistema jurídico y, al estar contenidos en la Constitución, funcionan como límites al legislador. En efecto, la legislación se encuentra sometida tanto formal como materialmente a la Constitución, en especial a su parte dogmática. Esto significaría el tránsito de un Estado legal a un Estado constitucional, donde las normas jurídicas no sólo están sometidas a un juicio de vigencia sino también a uno de validez. Y ésta no es solo una cuestión formal sino sustancial, en cuanto a que valora la coherencia de la ley con las normas constitucionales. Por eso se habla, como se decía líneas arriba, de que se está no solo ante una mera legalidad sino una estricta legalidad.

Uno de los más famosos temas que ha acarreado la importancia de los derechos fundamentales en el neoconsticionalismo es el referente a la relación entre derecho y moral, pues se considera, en términos generales, que la división y separación categóricas ya no es procedente. La defensa del legalismo ético se considera, en términos de Bobbio (1992), como un positivismo ideológico ${ }^{4}$ que después de lo acontecido durante la Segunda Guerra Mundial ya no responde a los nuevos estados democráticos y constitucionales. Esto porque la relación entre moral y derecho ya no se responde a través de una separación total, sino de considerar que si bien deben distinguirse no pueden estar incomunicados. Lo que lleva a considera que los derechos humanos son incorporar al sistema jurídico contenidos morales que son la finalidad del propio Derecho.

En cuanto a la discusión sobre cómo deben estructurarse los derechos fundamentales en la Constitución para su mejor funcionamiento en el sistema jurídico, suelen darse tres modelos: como principios, como reglas, o de forma mixta. Esto tiene que ver, en gran parte, con una teoría de la norma.

Existen tres posiciones básicas respecto a los tipos de normas jurídicas. En primer lugar, la que sostiene que existen dos tipos de normas jurídicas, a saber, los principios y las reglas, y que se pueden establecer criterios claros y definitivos para diferenciarlos (tesis fuerte de la separación). En segundo lugar, la que sostiene que si bien existen los dos tipos de normas, la determinación no puede darse directamente sobre el texto constitucional, sino en función de cómo se construye la norma jurídica por parte del intérprete; es decir, que existe una diferencia entre lo que es el texto normativo y la norma jurídica, pues de aquél pueden generarse diversas normas jurídicas en función del trabajo del intérprete $\mathrm{y}$, por lo tanto, puede generar tanto reglas como principios (tesis débil de la separación). Y, en tercer lugar, la que sostiene que no existe tal distinción y que, además, no es necesaria (tesis de la conformidad).

Las teorías neoconstitucionales son, en general, teorías principialistas al asumir la primera o segunda posición arriba mencionada. Es obvio que negar la distinción entre reglas y principios, afirmando que toda norma jurídica es una regla, imposibilitaría hablar de una teoría principialista y, por lo tanto, no habría discusión sobre si los derechos fundamentales deben construirse como reglas o como principios. Por lo tanto, se asume que, ya sea de manera fuerte o débil -cuestión que no se profundizará- se puede hablar de la existencia de dos tipos de normas jurídicas: los principios y las reglas.

Se han establecido diversos criterios para distinguir

3 El término "neoconstitucionalismo" se refiere a un conjunto de corrientes del pensamiento jurídico que intentan renovar el constitucionalismo clásico nacido de las revoluciones burguesas del siglo XVIII. Entre otros aspectos, destaca la importancia que tiene la efectividad de los derechos humanos en un sistema jurídico constitucional, así como una visión amplia del contenido de estos derechos - liberales, sociales, individuales, colectivos, etc.- (véase Prieto Sanchís, 2003: 101-135).

4 Legalismo ético se refiere a la posición que defiende que el cumplimiento de la ley, por el puro hecho de la ley, es un acto moral. Por lo tanto, el Derecho sólo puede garantizar por si mismo un valor: la seguridad jurídica. Los demás valores que pueden estar presentes en el contenido de una norma jurídica dependen de las discusiones políticas, de la decisión de las mayorías, pero no tienen que ver con el Derecho en sí mismo. 
las reglas y los principios. En este trabajo, se explicarán algunos de ellos, pero se advierte que ninguno es completo ni suficiente para distinguir con claridad las reglas y los principios, pero son importantes pues de ellos dependerá la manera en que el intérprete generará la norma nacida del texto jurídico: si como principio o como regla.

Tres de las diferencias más clásicas para distinguir principios y reglas son "el carácter hipotéticocondicional", el "modo final de aplicación", y el "modo final de aplicación" (Ávila, 2011, pp. 58-70). Se hará un breve comentario de cada uno.

- El carácter hipotético-condicional: Según este criterio, las reglas están construidas con un carácter de "Si... entonces", "Si A entonces B", donde claramente se establecen el supuesto y la consecuencia y, por lo tanto, si se cumple en los hechos el contenido del antecedente se debe cumplir el consecuente. Por ejemplo, "Quien priva de la vida a otro (A) comete el delito de homicidio y tendrá una pena de 5 a 10 años de prisión (B)". En cambio, los principios no tienen esta estructura y más bien son solo la descripción de una situación ideal; es decir, una estructura de "Debe ser C". Por ejemplo, "el hombre y la mujer son iguales ante la ley".

- El criterio de "modo final de aplicación": Según este criterio, las reglas se aplican a través de un silogismo, de un juicio de subsunción. Es decir, si la estructura de la regla es "Si A entonces B", se debe comprobar que en los hechos se actualiza la hipótesis A, y por lo tanto debe darse la consecuencia. Por ejemplo, "Quien priva de la vida a otro comete el delito de homicidio y tendrá una pena de 10 años de presión". "Juan mató a Pedro". "Entonces Juan cometió el delito de homicidio y tendrá 10 años de prisión". En cambio, los principios requieren de un trabajo argumentativo que justifique su aplicación al caso concreto, y que implica un juicio de ponderación.

- El criterio del conflicto normativo: Este es tal vez el más conocido, debido a que ha cobrado gran importancia en la teoría de los derechos fundamentales. El conflicto normativo puede darse en abstracto y en concreto. El primero se refiere a "un conflicto conceptual que se produce cada vez que dos normas conectan dos consecuencias jurídicas incompatibles (y por lo tanto ofrecen dos soluciones incompatibles) a dos clases de hechos jurídicos" (Serpe, 2010, p. 48). Es decir, este conflicto no requiere de un hecho concreto que actualice el supuesto normativo, y por lo tanto esas reglas no pueden "convivir" en el sistema jurídico, ya que se entiende que éste es coherente. Por su parte, el conflicto en concreto aparece en la fase de aplicación de las normas jurídicas a un caso concreto, que "en abstracto" no se muestras como contradictorias. Supuesto esto, se afirma que el conflicto normativo de las reglas se da en abstracto y que, por lo tanto, una de las reglas en conflicto debe quedar sin validez o contener una excepción que supere el conflicto, y así salvar la coherencia del sistema jurídico. En cambio, el conflicto normativo de los principios se da en concreto, pues es necesario un caso concreto donde se dé cuenta que dos o más principios, que pueden convivir sin problemas en abstracto en el sistema jurídico, están en conflicto.

Estas tres diferencias entre principios y reglas han sido la base de análisis más profundos al respecto. Se trata de análisis que sostienen las limitaciones de dichos criterios y la necesidad de establecer criterios más sólidos. Se verán unos casos.

Robert Alexy es uno de los exponentes más conocidos de la teoría principialista, a partir de su obra Theorie der Grundrechte ("Teoría de los derechos fundamentales"), publicada en 1985. En ella analiza la estructura de las normas consideradas derechos fundamentales, y establece que las normas pueden dividirse en dos clases: principios y reglas (Alexy, 1993). Alexy se inclinaría por la postura que considera la separación de principios y reglas de manera fuerte, pues para él existe un criterio que permite distinguir con toda precisión entre reglas y principios: los principios son mandatos de optimización, y las reglas son normas que pueden ser cumplidas o no.

Para Alexy, los principios son normas jurídicas que "ordenan que algo sea realizado en la mayor medida posible, dentro de las posibilidades jurídicas y reales existentes" (Alexy, 1993, p. 86). Entonces, los principios son mandatos de optimización, que pueden cumplirse en diferente grado y que su cumplimiento no sólo depende de las posibilidades reales sino también de las jurídicas; en este sentido, las posibilidades jurídicas se determinan por principios y reglas opuestos al principio en cuestión. Por su parte, las reglas son normas que solo pueden ser cumplidas o no; son del todo o nada. Si una regla es válida entonces debe de hacerse exactamente lo que ella exige, ni más ni menos. Por lo tanto, las reglas contienen determinaciones en el ámbito de lo fáctico y jurídicamente posible (Alexy, 1993, pp. 6768).

Otra diferencia consiste en el carácter prima facie que existe entre las reglas y los principios. Los 
principios al establecer que algo sea realizado en la mayor medida de lo posible, teniendo en cuanta las posibilidades jurídicas y fácticas, no es un mandato definitivo sino sólo prima facie (Alexy, 1993, p. 99), es decir, que en un primer momento los principios mandan realizar algo, pero en un segundo momento su grado de cumplimiento será diferente según el caso concreto. En cambio, las reglas, al ser mandatos definitivos, contienen una determinación considerando las posibilidades jurídicas y fácticas, y si cuenta con imposibilidades de este tipo entonces será una regla inválida, de lo contrario contará con validez definitiva. Ahora bien, esta validez puede perderse con cláusulas de excepción.

Un análisis también interesante sobre los criterios diferenciadores es el realizado por Humberto Ávila (2011, p. 64-70). Después de criticar las limitaciones de los criterios tradicionales, propone una diferenciación heurística. De hecho, él se ubica en la tesis débil de la separación, pues su criterio no se basa principalmente en el texto normativo en sí mismo, sino en la manera que el intérprete genera la norma jurídica; por eso se admite la coexistencia de principios y reglas en razón de una misma disposición. Para ello establece tres criterios diferenciadores:

1. Criterio de la naturaleza del comportamiento descrito: Este criterio se basa en el hecho de que tanto los principios como las reglas, describen un comportamiento que se debe de adoptar. En este sentido, las reglas son normas inmediatamente descriptivas, pues establecen obligaciones, permisos y prohibiciones mediante la descripción de la conducta a ser adoptada.Porsuparte, los principios sonnormas inmediatamente finalistas, pues establecen un estado de cosas que requiere adoptar ciertos comportamientos para su realización. Al ser normas jurídicas, tanto principios como reglas establecen un deber a cumplir. Pero por la manera de describir el comportamiento, los deberes mediato e inmediato son diferentes en cada tipo de norma. Así, el deber inmediato para los principios es la promoción del estado ideal de las cosas, mientras que para las reglas es la adopción de las conductas descritas. Y el deber mediato para las reglas es adoptar la conducta necesaria para lograr el estado ideal de las cosas, y el de las reglas es mantener la fidelidad a la finalidad subyacente y a los principios superiores que reportan a la regla.

2. Criterio de la naturaleza de la justificación exigida: Este criterio se refiere a las exigencias de argumentación que impone cada tipo de norma. La aplicación de las reglas requiere evaluar la correspondencia entre la construcción de los hechos y la construcción de la norma y de la finalidad que le da soporte. En cambio, la aplicación de los principios exige evaluar la correlación entre las consecuencias de la conducta considerada necesaria y la realización del estado de cosas puesto como fin.

3. Criterio de la medida de contribución a la decisión: Este criterio se refiere a la pretensión que tiene la norma de aportar a la decisión sobre el caso concreto. Los principios consisten en normas primariamente complementarias y preliminarmente parciales, en la medida en que, además de comprender sólo parte de los aspectos relevantes para una toma de decisión, no tienen la pretensión de generar una solución específica, sino de contribuir, junto a otras razones, a la toma de decisión.

Estos criterios de diferenciación conducen a Ávila a establecer las siguientes definiciones de principios y reglas:

Las reglas son normas inmediatamente descriptivas, primariamente retrospectivas $\mathrm{y}$ con pretensión de decidibilidad y comprensión, para cuya aplicación se exige la valoración de la correspondencia, siempre centrada en la finalidad que les da soporte o en los principios axiológicamente superiores, entre la construcción conceptual de la descripción normativa y la construcción conceptual de los hechos.

Los principios son normas inmediatamente finalistas, primariamente prospectivas y con pretensión de complementariedad y parcialidad, para cuya aplicación se requiere una valoración de la correlación entre el estado de cosas que debe ser promovido y los efectos derivados de la conducta considerada necesaria para su promoción. (Ávila, 2011, p. 70)

Aunque con diferencias, la posición de Alexy y Ávila son cercanas en el sentido de que comprenden los principios como mandatos a construir o realizar un estado deseado. Ya sea por considerarlos como mandatos de optimización o como normas inmediatamente finalistas. Siendo así, finalmente, es necesario un trabajo de interpretación y argumentación que posibilite la aplicación del principio, que sin duda es diferente al de la aplicación de la regla.

En cuanto a la construcción de los derechos fundamentales, Alexy se pregunta cuál es el mejor modelo, y analiza las ventajas y desventajas de construir las normas como principios o como reglas; para él, ésta es una cuestión importantísima, pues sin dicha distinción no puede existir una teoría adecuada de los límites, ni una teoría satisfactoria de la colisión 
y tampoco una teoría completa acerca del papel que tienen los derechos fundamentales en el sistema jurídico.

A partir de ese supuesto, Alexy separa las formas de aplicación de los principios en tres modelos. El primer modelo sería el "puro de principios", donde las normas iusfundamentales serían principios y las reglas serían el resultado de ponderaciones. Sobre él, Alexy señala que sería "una 'ilusión' pensar que el problema de los límites del derecho fundamental puede ser solucionado a través de las disposiciones sobre reservas y restricciones que se encuentran en la Ley Fundamental" (Alexy, 1993, p. 96). Al segundo le llama "modelo puro de reglas", donde las normas de derechos fundamentales deben estar libres de ponderación; en este caso se plantearían los derechos fundamentales en tres tipos: sin reserva alguna, con reserva simple y con reserva cualificada. Sobre este, en general, Alexy considera que puede en unos casos proteger poco y en otro demasiado el contenido de los derechos. El tercer modelo, al que se suscribe Alexy, es el mixto, que surge de la vinculación de un nivel de principios con un nivel de reglas. En este sentido, en la mayoría de las Constituciones de los estados que se adscriben al neoconstitucionalismo se encuentra el modelo mixto; es decir, una construcción de derechos fundamentales tanto como principios como reglas. En general, en la Constitución mexicana, los derechos fundamentales están establecidos como reglas y como principios.

\section{LA INTERACCIÓN ENTRE BIOÉTICA Y DERECHOS HUMANOS}

En el campo de la bioética no es ajeno hablar de principios. De hecho, ha predominado el pensamiento bioético que se funda en principios, que más o menos suele enunciar cuatro: principio de autonomía, principio de no-maleficiencia, principio de beneficiencia, y principio de justicia. Por supuesto que estos principios responden a una tradición bioética concreta -vinculada con la cultura occidental-, y que se puede hablar de principios bioéticos diferentes en otras tradiciones (Siurana, 2010). Pero lo que en este momento se destaca es el uso de principios por parte de la bioética que deben aplicarse a cada caso concreto para tomar una decisión.

protegidas por un claro campo de autonomía, no pueden ser sostenidas si colocan en riesgo la salud del menor. En estos casos está justificado intervenir en la autonomía familiar con el objeto de impedir una afectación a la integridad del menor. Ello obedece a que los derechos parentales tienen fundamento precisamente en la protección que deben brindar los padres a sus niños. De manera similar, la libertad religiosa y el derecho a la vida privada familiar no comprenden la imposición de prácticas religiosas que comprometan la salud y vida de los niños. En otras palabras: la libertad religiosa no confiere a los padres la autoridad para decidir sobre la vida o la muerte de sus hijos menores de edad; así, los derechos de los padres encuentran su límite ahí donde se pone en riesgo la vida de sus hijos. (Registro: 2019242).

Como se observa, para establecer este criterio, la Primera Sala desarrolló el contenido de por lo menos cuatro principios: derecho a la salud, derecho a la vida, derecho a la privacidad familiar y derecho a la libertad religiosa. Estableció un conflicto de principios entre los derechos del menor -vida y salud-y los derechos de los padres - privacidad y libertad-, para determinar que en caso de estar en riesgo la vida y la salud del menor, el Estado puede restringir justificadamente la privacidad familiar y la libertad religiosa.

En el siguiente criterio, también la Primera Sala de la SCJN determinó que el consentimiento informado del paciente es una conducta que cumple con los derechos a la vida, a la integridad física y a libertad de conciencia:

El consentimiento informado es consecuencia necesaria o explicitación de derechos a la vida, a la integridad física y a la libertad de conciencia, el cual consiste en el derecho del paciente de otorgar o no su consentimiento válidamente informado en la realización de tratamientos o procedimientos médicos. En tal sentido, para que se pueda intervenir al paciente, es necesario que se le den a conocer las características del procedimiento médico, así como los riesgos que implica tal intervención. A través de éste el paciente asume los riesgos y consecuencias inherentes $o$ asociados a la intervención autorizada; pero no excluye la responsabilidad médica cuando exista una actuación negligente de los médicos o instituciones de salud involucrados. (Registro: 2001271).

con el derecho al concretizar la conducta que adecuadamente cumple con la finalidad enunciada en las normas de derechos humanos.

\section{CONCLUSIÓN}

Una de las relaciones que tiene la bioética con el derecho es el desarrollo del contenido de los derechos humanos. Como se ha explicado, al estar los derechos humanos plasmados como principios, su aplicación requiere de un trabajo argumentativo que posibilite determinar cuál es la conducta que optimiza el logro del fin señalado en la norma. Cuando se trata de una situación que implica a la bioética, el operador del derecho requiere forzosamente un diálogo con ella 
para poder determinar, desde los principios que establecen los derechos humanos, la conducta que optimice su cumplimiento.

En este sentido, no sólo la aplicación del derecho a la salud tiene que ver con la bioética, pues otros derechos humanos también pueden estar implicados en este ámbito. Así, por ejemplo, pueden implicar un contenido bioético los siguientes derechos: al libre desarrollo de la personalidad, a la libertad de tránsito, a la libertad religiosa, a la educación, a la libertad reproductiva, de las personas con discapacidad, de la infancia, de las personas adultas mayores, a la igualdad y no-discriminación, entre otros.

\section{BIBLIOGRAFÍA}

Alexy, Robert (1993). Teoría de los derechos fundamentales, Madrid: Centro de Estudios Constitucionales.

Ávila, Humberto (2011). Teoría de los principios, Madrid: Marcial Pons.

Bobbio, Norberto, El problema del positivismo jurídico, Fontamara, México, 1992.

Ferrajoli, Luigi (2009). Derecho y razón. Teoría del garantismo penal, Madrid: Trortta.

Herrera Flores, Joaquín (2005). Los derechos humanos como productos culturales. Crítica del humanismo abstracto, Madrid: Catarata.

Hohfeld, Wensley Newcomb (1991). Conceptos jurídicos fundamentales, México: Fontamara.

Peces-Barba Martínez, Gregorio (1999). Curso de derechos fundamentales. Teoría general, Madrid: Universidad Carlos III de Madrid.

Prieto Sanchís, Luis (2003). Justicia constitucional y derechos fundamentales, Madrid: Trotta.

Ramírez García, Hugo Saúl et al. (2014). Derechos humanos, México: Oxford University Press.

Rosillo Martínez, Alejandro (2013). La fundamentación de derechos humanos desde América Latina, México: Ítaca.

Sánchez Rubio, David (2018). Derechos humanos instituyentes, pensamiento crítico y praxis de liberación, Madrid: Akal.

Serpe, Alessandro (2010). “Argumentando a partir de los derechos humanos. La ponderación en serio": Utopía y praxis latinoamericana. Revista Internacional de Filosofía Iberoamericana y Teoría Social, Universidad del Zulia, Año 15, no. 51.

Siurana Aparisi, Juan Carlos (2010). "Los principios de la bioética y el surgimiento de una bioética intercultural" en Veritas, $\mathrm{N}^{\circ} 22$, marzo, pp. 121157.

Época: Décima Época. Registro: 2000341. Instancia: Primera Sala. Tipo de Tesis: Aislada. Fuente: Semanario Judicial de la Federación y su Gaceta. Libro VI, Marzo de 2012, Tomo 1. Materia(s): Constitucional. Tesis: 1a. XLIV/2012 (10a.). Página: 274.

\section{JURISPRUDENCIA}

Poder Judicial de la Federación. Registro: 2019242.

Época: Décima Época. Instancia: Primera

Sala. Tipo de Tesis: Aislada. Fuente: Gaceta

del Semanario Judicial de la Federación.

Libro 63, febrero de 2019, Tomo I. Materia(s):

Constitucional. Tesis: 1a. IX/2019 (10a.). Página:

720 .

Poder Judicial de la Federación. Registro: 2001271. Época: Décima Época. Instancia: Primera Sala. Tipo de Tesis: Aislada. Fuente: Semanario Judicial de la Federación y su Gaceta. Libro XI, agosto de 2012, Tomo 1. Materia(s): Constitucional. Tesis: 1a. XLIII/2012 (10a.). Página: 478

Poder Judicial de la Federación. Registro: 2000341. Época: Décima Época. Instancia: Primera Sala. Tipo de Tesis: Aislada. Fuente: Semanario Judicial de la Federación y su Gaceta. Libro VI, Marzo de 2012, Tomo 1. Materia(s): Constitucional. Tesis: 1a. XLIV/2012 (10a.). Página: 274

Poder Judicial de la Federación. Registro: 165826. Época: Novena Época. Instancia: Pleno. Tipo de Tesis: Aislada. Fuente: Semanario Judicial de la Federación y su Gaceta. Tomo XXX, Diciembre de 2009. Materia(s): Constitucional. Tesis: P. LXVIII/2009. Página: 6 